\title{
BIOMARKERS SPECIFIC TO DENSELY-IONISING (HIGH LET) RADIATIONS
}

\author{
D. J. Brenner $\dagger$, N. Okladnikova $\ddagger$, P. Hande $\dagger$, L. Burak $\ddagger$, C. R. Geard $\dagger$ and T. Azizova $\ddagger$ \\ $†$ Center for Radiological Research, Columbia University \\ 630 West 168th Street, New York, NY 10032, USA \\ $\ddagger$ Southern Urals Biophysics Institute, Ozyorsk, Russia
}

\begin{abstract}
There have been several suggestions of biomarkers that are specific to high LET radiation. Such a biomarker could significantly increase the power of epidemiological studies of individuals exposed to densely-ionising radiations such as alpha particles (e.g. radon, plutonium workers, individuals exposed to depleted uranium) or neutrons (e.g. radiation workers, airline personnel). We discuss here a potentially powerful high LET biomarker (the H value) which is the ratio of induced interchromosomal aberrations to intra-arm aberrations. Both theoretical and experimental studies have suggested that this ratio should differ by a factor of about three between high LET radiation and any other likely clastogen, and will yield more discrimination than the previously suggested $\mathrm{F}$ value (ratio of inter-chromosomal aberrations to intra-chromosomal inter-arm aberrations). Evidence of the long-term stability of such chromosomal biomarkers has also been generated. Because these stable intra-arm and interchromosomal aberrations are (1) frequent and (2) measurable at long times after exposure, this $\mathrm{H}$ value appears to be a practical biomarker of high LET exposure, and several in vitro studies have confirmed the approach for unstable aberrations. The approach is currently being tested in a population of Russian radiation workers exposed several decades ago to high- or low LET radiation.
\end{abstract}

\section{INTRODUCTION}

Home occupants exposed to radon, plutonium workers, clean-up workers, nuclear energy research workers, well loggers, and airline flight personnel, have all potentially been exposed to significant doses of densely-ionising (high LET) radiation, such as alpha particles or neutrons. In occupational exposure settings, the high LET radiation exposure is often combined with exposure to low LET radiations (e.g. gamma rays, tritium), as well as chemical carcinogens or clastogens.

Another area in which a high LET radiation exposure is of potential significance is gamma ray risk estimation in the atomic bomb survivors at Hiroshima. Because the neutron component of the overall dose is not well known ${ }^{(1)}$, there is considerable uncertainty concerning the proportion of the excess cancer risk which is attributable to neutrons - which in turn may affect the risk estimates from gamma rays. Previous attempts to apply LET-specific biomarkers to this particular problem have met with only limited success ${ }^{(2-5)}$.

Consequently there has been much interest in finding a detectable biological 'fingerprint' preferentially produced by high LET radiation. An expert report on methods to arrive at risk estimates for radiation-induced human cancer $^{(6)}$ suggested that identification of a radiation 'signature' was a key research need.

Several experimental observations have been made suggesting biological lesions that may be uniquely or preferentially produced by high LET radiation ${ }^{(7-10)}$, but these have no known mechanistic basis, and other results have not confirmed the initial suggestions ${ }^{(11-14)}$. This lack of a mechanistic background inevitably leads

Contact author E-mail: djb3@columbia.edu to uncertainties about the general applicability of the proposed heuristic markers ${ }^{(15-17)}$. Here, we focus on a preferential effect that is expected mechanistically, and appears to be observed experimentally.

Exposure to ionising radiation produces chromosome aberrations. An important aberration formation mechanism is pairwise interaction of two elementary damage sites - often, though not necessarily, identified as double-strand breaks - in which ends from different breaks meet and join in an illegitimate recombination. These exchange-type aberrations (see Figure 1 and Table 1) can be inter-chromosomal if the double-strand breaks are on different chromosomes, or intra-chromosomal inter-arm if the double-strand breaks are on dif-

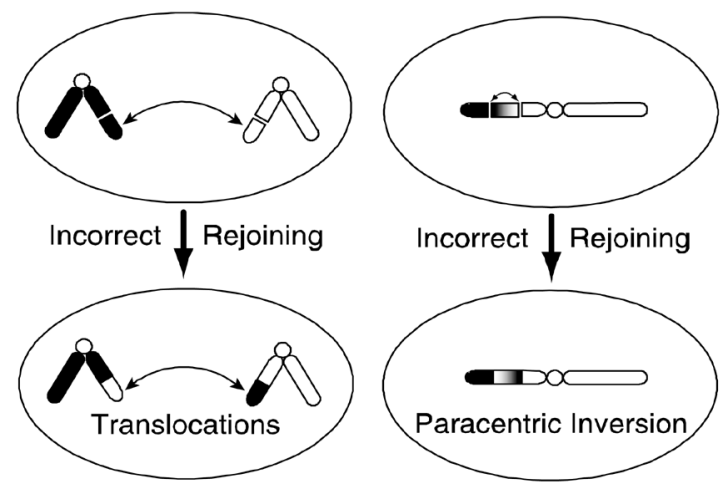

Figure 1. Schematic illustration of the production of interchromosomal (left panel) and intra-chromosomal intra-arm aberrations (right panel), in this case for stable aberrations. The $\mathrm{H}$ value (see Table 1) is the ratio of the number of induced inter-chromosomal aberrations (translocations or dicentrics) to intra-arm aberrations (paracentric inversions or interstitial deletions). 


\section{J. BRENNER, N. OKLADNIKOVA, P. HANDE, L. BURAK, C. R. GEARD and T. AZIZOVA}

ferent arms of the same chromosome, or intra-chromosomal intra-arm if the double-strand breaks are on one chromosome arm.

The chance that two double-strand breaks come close enough to undergo an exchange depends on their relative initial location and relative motion. In fact pairs of breaks that are close to one another are more likely to interact than pairs that are comparatively far apart. Because individual chromosomes are localised in domains that are smaller than the cell nucleus, this increased interaction probability of nearby breaks is expected to produce a bias towards intra-arm compared with inter-chromosomal aberrations.

However, high LET radiations such as alpha particles produce energy depositions, and thus chromosome breaks, that are, on average, much closer together than those produced by chemical clastogens or gamma rays. Thus at equal damage levels, high LET radiation is much more likely to produce multiple breaks within a given chromosome than are chemical clastogens or gamma rays, and so yields of intra-chromosomal aberrations are even further increased relative to interchromosomal aberrations; thus the resulting smaller $\mathrm{H}$ value is a potential 'fingerprint' of prior exposure to densely-ionising radiations. $\mathrm{H}$ values in stable aberrations can, in principle, be measured in the cells (and their progeny) of an individual many years after exposure.

Previous studies on ratios of chromosomal aberrations as biomarkers of high LET damage focused on the ratio of inter-chromosomal to intra-chromosomal inter-arm aberrations. This is known as the $\mathrm{F}$ value (see Table 1). Studies on the use of this marker were suggestive that there was a difference between low and high LET F values, but that current FISH technology is not adequate to consistently observe this in an epidemiological setting ${ }^{(5,18-25)}$.

On average, however, the breaks underlying intrachromosomal intra-arm aberration formation would be even closer together than those producing intra-chromosomal inter-arm aberrations, so intra-arm aberration yields would be subject to even more proximity effects than inter-arm yields. This is the rationale for predicting that the $\mathrm{H}$ value (inter-chromosomal vs. intra-chromo- somal intra-arm, see Table 1) would have a stronger LET dependency than would the F value.

\section{METHODS}

Mechanistic models to predict the ratio of interchromosomal to intra-chromosomal (inter-arm and/or intra-arm) aberrations have been discussed by several groups $^{(18,19,24,26)}$. For example, using a polymer description for large-scale chromatin geometry and using realistic models for double-strand break motion, we have made theoretical estimates for size distributions of intrachanges at low vs. high $\mathrm{LET}^{(26)}$. The ratio of interchromosomal to intra-arm intrachanges was derived from the size distribution and predictions were made of the trends of the ratio of inter-chromosomal to intra-arm aberrations at low vs. high LET. Because inversions are more frequent than estimates of randomness would indicate, and are transmissible to daughter cells, the ratio $(\mathrm{H})$ of stable inter-chromosomal to intra-arm aberrations could be a practical mechanistically-based 'fingerprint' of past exposure to high LET radiation.

Most of the initial data on $\mathrm{H}$ values (see below) has come from using standard cytometric techniques to score unstable aberrations. While measuring unstable aberrations is of use for in vitro-based studies, their relatively short half life in vivo makes them impractical as a 'fingerprint' of high LET exposure many years after the exposure. Thus it is important to be able to measure the stable version of the $\mathrm{H}$ value (translocations/ paracentric inversions, see Table 1).

Translocations can be measured using standard techniques ${ }^{(27)}$, involving composite DNA probes specific to several large chromosomes. Until recently, however, FISH probes were not available for paracentric inversions. Because standard FISH probes as well as the more recent 'spectral karyotyping' (SKY) and 'M-FISH' techniques ${ }^{(28,29)}$, paint all of a given chromosome in the same colour, they cannot analyse intrachromosomal rearrangements. However, a newer FISH technique has recently become available yielding high resolution multi-colour banding ${ }^{(30)}$. A high-resolution technique which has just become commercially available (M-BAND, MetaSystems, Germany) is based on

Table 1. Relevant chromosome aberration types and ratios.

\begin{tabular}{llcc}
\hline & Inter-chromosomal & $\begin{array}{c}\text { Intra-chromosomal } \\
\text { inter-arm }\end{array}$ & $\begin{array}{c}\text { Intra-chromosomal } \\
\text { intra-arm }\end{array}$ \\
\hline Stable (non-lethal) & Translocation & Pericentric inversion & Paracentric inversion \\
Unstable (lethal) & Dicentric & Centric ring & Interstitial deletion \\
F value: & vs. & value:
\end{tabular}




\section{BIOMARKERS SPECIFIC TO DENSELY-IONISING RADIATION}

region-specific partial chromosome paints combined with quantitative colour ratio analysis. Each partialchromosome paint is labelled using a unique fluorochrome combination and partly overlaps with its neighbouring one, along a chromosome. The resulting fluorescence intensity pattern along the chromosome axis shows a continuous change of colour ratios. Pseudo colours are assigned to chromosome sections of similar colour ratios, giving rise to a reproducible colour banding pattern that does not depend on chromosome condensation. The colour ratio analysis effectively multiplies the initial resolution of the probe by a factor of 3 to 4. For a given number of colour bands, the complexity of the probe cocktail as well as the number of fluorochrome combinations is about three times less with this approach than with the 'colour bar code' approach using individually labelled $\mathrm{YACs}^{(31)}$. For example, for chromosome 5, seven partial chromosome paints are used and 25 colour bands are achieved.

\section{RESULTS}

\section{Stability of ratios of chromosome aberrations}

It is important to distinguish between the stability over time of the yields of individual aberrations (such as translocations), and the stability of the ratio of aberrations, which is the endpoint of interest here. Of relevance here is the stability of the ratio of inter-chromosomal to intra-chromosomal aberrations. Although we have no direct evidence of the stability of the $\mathrm{H}$ value over long periods, data on the stability of the $\mathrm{F}$ value (see Table 1) are available. Over a period of more than 20 years, there have been four measurements of yields of translocations and pericentric inversions in samples of peripheral lymphocytes taken from a total of over 400 exposed survivors of the Hiroshima explosion, as well as controls.

The first study, reported by Awa et $a l^{(32)}$, examined blood samples taken in 1968 and 1969; the second study, by Ohtaki ${ }^{(33)}$, examined blood samples taken between 1977 and 1991; the third study, by Sachs et $\mathrm{al}^{(34)}$, examined blood samples drawn during 1989 and 1990; the fourth study, by Kodama et $a l^{(5)}$, examined blood samples drawn between 1977 and 1998. The $F$ values from the results of the four studies were respectively $6.8 \pm 0.4$ (23 to 24 years post exposure $\left.{ }^{(32)}\right)$, $5.7 \pm 0.4$ (32 to 46 years post exposure $\left.{ }^{(33)}\right), 6.2 \pm 0.7$ (44 to 45 years post exposure ${ }^{(34)}$ ) and $7.2 \pm 0.2$ (32 to 53 years post exposure $\left.{ }^{(5)}\right)$. The consistency of these values suggests that $F$ values do not change significantly over very long periods of time, though one cannot rule out a change in the $\mathrm{F}$ value between the time of the explosions (1945) and the first of these analyses in 1968.

If the $\mathrm{F}$ value is indeed stable over long periods, it seems likely that the $\mathrm{H}$ value would have a similar characteristic.

\section{Measured $H$ values as a function of LET}

As discussed above, $\mathrm{H}$ values for unstable aberrations can be measured using standard cytometric techniques. Subsequent to our initial theoretical predictions, $\mathrm{H}$ values for low vs. high LET were measured by Bauchinger and Schmid ${ }^{(21)}$ in Munich (ratio of low LET:high LET $H$ values $\approx 3 \pm 1$ ), by Deng et al ${ }^{(25)}$ at Livermore (ratio of low LET:high LET $\mathrm{H}$ values $\approx 1.9 \pm 0.5$ ), by Anderson et $\mathrm{el}^{(35)}$ at Harwell (ratio of low LET:high LET $\mathrm{H}$ values $\approx 4$ ), and by our own group ${ }^{(36)}$ (ratio of low LET:high LET H values $\approx 1.5 \pm 0.3$ ). Some typical results (from the Munich group) are shown in Figure 2, showing a clear separation between low and high LET.

\section{CONCLUSIONS}

There is a need for a biomarker that could distinguish between damage induced by high LET radiations, such as alpha particles and neutrons, and damage induced by other carcinogens or clastogens. Such a biomarker would have the potential to facilitate 'molecular radiation epidemiology', analogous to the development of molecular epidemiology with DNA adducts ${ }^{(37)}$.

As discussed above, various empirical (i.e. nonmechanistically based) biomarkers for past high LET exposure have been suggested, but subsequent analyses have not confirmed these suggestions. Besides the ratios of aberrations discussed here, one other mechanisticallybased endpoint that does have potential is yields of complex chromosome aberrations ${ }^{(35)}$; this endpoint may well be useful for analysis immediately or soon after exposure, but the complex aberrations are largely nontransmissible (in vitro, exposure to 0.5 Gy of alpha particles produced a $5 \%$ yield of complex aberrations at

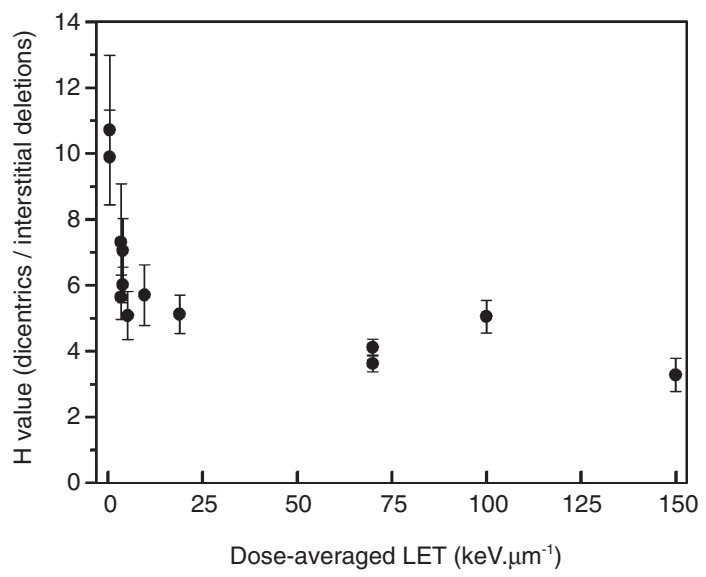

Figure 2. Measured ratio of dicentric aberration to interstitial deletion yields (the $\mathrm{H}$ value for unstable aberrations), as a function of the dose-averaged LET of the incident radiation. Note the clear separation between values at low and high LET. Data derived from the work of Bauchinger and Schmid ${ }^{(21)}$. 


\section{J. BRENNER, N. OKLADNIKOVA, P. HANDE, L. BURAK, C. R. GEARD and T. AZIZOVA}

first mitosis, but only a $0.25 \%$ yield by the third mitosis ${ }^{(35)}$ ), which probably makes them impractical as a long-term biomarker. By contrast, the stable aberrations under discussion here have much higher transmissabilities.

Earlier studies on ratios of chromosomal aberrations focused on the ratio of inter-chromosomal to intrachromosomal inter-arm aberrations, the $\mathrm{F}$ value (see Table 1). Studies suggested that there was a difference between low and high LET F values, but that current FISH technology is too inaccurate/imprecise to observe this difference consistently in an epidemiological setting ${ }^{(18-25)}$. There is a theoretical rationale for predicting that the $\mathrm{H}$ value, involving intra-arm aberrations (see Table 1) would have a stronger LET dependency than would the $\mathrm{F}$ value.

Based on the data available to date it seems likely that high LET radiation does produce uniquely low $\mathrm{H}$ values, which thus represents a biomarker with a firm theoretical basis. Practically, the technology for measuring paracentric inversions remains in its infancy, and so more work is needed to evaluate its potential. The feasibility of using this biomarker in epidemiological studies is currently under test in a study of workers from the Mayak nuclear industrial complex ${ }^{(38)}$, who were exposed several decades ago to plutonium or to gamma rays, or to both.

\section{ACKNOWLEDGEMENTS}

Helpful advice from Drs Rainer Sachs, Ruth Neta, and James Tucker is gratefully acknowledged. This work was supported by grants DE-FC03-01EH01002 from the U.S. Department of Energy, and by Grants RR11623, ES-07361 and CA-49062 from the U.S. National Institutes of Health.

\section{REFERENCES}

1. Straume, T., Egbert, S. D., Woolson, W. A., Finkel, R. C., Kubik, P. W., Gove, H. E., Sharma, P. and Hoshi, M. Neutron Discrepancies in the DS86 Hiroshima Dosimetry System. Health Phys. 63, 421-426 (1992).

2. Tatsumi, J. Dose Estimation of Radiation-Exposed People by ESR. Film-Badge News (Tokyo) 125, 1-10 (1986).

3. Brenner, D. J. Direct Biological Evidence for a Significant Neutron Dose to Survivors of the Hiroshima Atomic Bomb. Radiat. Res. 145, 501-507 (1996).

4. Schmidt, E. and Bauchinger, M. Comments on "Direct Biological Evidence for a Significant Neutron Dose to Survivors of the Hiroshima Atomic Bomb” by D.J. Brenner. Radiat. Res. 146, 479-482 (1996).

5. Kodama, Y., Ohtaki, K., Awa, A. A., Nakano, M., Itoh, M. and Nakamura, N. The F Value for Chromosome Aberrations in Atomic Bomb Survivors does not Provide Evidence for a Primary Contribution of Neutrons to the Dose in Hiroshima. Radiat. Res. 152, 558-562 (1999).

6. Report on a Workshop to Examine Methods to Arrive at Risk Estimates for Radiation-Induced Cancer in the Human, Based on Laboratory Data. Radiat. Res. 135, 434-437 (1993).

7. Savage, J. R. K. and Holloway, M. Induction of Sister-Chromatid Exchanges by d(42 MeV)-Be Neutrons in Unstimulated Human-Blood Lymphocytes. Br. J. Radiol. 61, 231-234 (1988).

8. Vahakangas, K., Samet, J., Metcalf, R., Welsh, J., Bennett, W., Lane, D. and Harris, C. Mutation of p53 and ras Genes in Radon-Associated Lung Cancer from Uranium Miners. Lancet 339, 576-580 (1992).

9. Taylor, J. A., Watson, M. A., Devereux, T. R., Michels, R. Y., Saccamanno, G. and Anderson, M. p53 Mutation Hotspot in Radon-Associated Lung Cancer. Lancet 343, 86-87 (1994).

10. Lucas, J. N. Cytogenetic Signature for Ionizing Radiation. Int. J. Radiat. Biol. 73, 15-20 (1998).

11. Shubber, E. K. and al-Shaikhly, A. W. Cytogenetic Analysis of Blood Lymphocytes from X-Ray Radiographers. Int. Arch. Occup. Environ. Health 61, 385-389 (1989).

12. McDonald, J. W., Taylor, J. A., Watson, M. A., Saccomanno, G. and Devereux, T. R. p53 and K-ras in Radon-Associated Lung Adenocarcinoma. Cancer Epidemiol. Biomarkers Prev. 4, 791-793 (1995).

13. Hollstein, M., Bartsch, H., Wesch, H., Kure, E. H., Mustonen, R., Muhlbauer, K. R., Spiethoff, A., Wegener, K., Wiethege, T. and Muller, K. M. p53 Gene Mutation Analysis in Tumors of Patients Exposed to Alpha-Particles. Carcinogenesis 18, 511-516 (1997).

14. Wu, H., George, K. and Yang, T. C. Estimate of the Frequency of True Incomplete Exchanges in Human Lymphocytes Exposed to $1 \mathrm{GeV/u}$ Fe Ions in vitro. Int. J. Radiat. Biol. 75, 593-599 (1999).

15. Peto, J. and Darby, S. Lung Cancer. Radon Risk Reassessed. Nature 368, 97-98 (1994).

16. Venitt, S. and Biggs, P. J. Radon, Mycotoxins, p53, and Uranium Mining. Lancet 343, 795 (1994).

17. Hei, T. K., Bedford, J. and Waldren, C. A. p53 Mutation Hotspot in Radon-Associated Lung Cancer. Lancet 343, 11581159 (1994).

18. Brenner, D. J. and Sachs, R. K. Chromosomal Fingerprints of Prior Exposure to Densely-Ionizing Radiation. Radiat. Res. 140, 134-142 (1994).

19. Savage, J. R. K. and Papworth, D. G. Comment on the Ratio of Chromosome-Type Dicentric Interchanges to Centric Rings for Track-Clustered Compared with Random Breaks. Radiat. Res. 146, 236-240 (1996). 


\section{BIOMARKERS SPECIFIC TO DENSELY-IONISING RADIATION}

20. Schwartz, J. L. and Hsie, A. W. Genetic and Cytogenetic Markers of Exposure to High-Linear Energy Transfer Radiation. Radiat. Res. 148, S87-92 (1997).

21. Bauchinger, M. and Schmid, E. LET Dependence of Yield Ratios of Radiation-Induced Intra- and Interchromosomal Aberrations in Human Lymphocytes. Int. J. Radiat. Biol. 74, 17-25 (1998).

22. Nakamura, N., Tucker, J. D., Bauchinger, M., Littlefield, L. G., Lloyd, D. C., Preston, R. J., Sasaki, M. S., Awa, A. A. and Wolff, S. F Values as Cytogenetic Fingerprints of Prior Exposure to Different Radiation Qualities: Prediction, Reality and Future. Radiat. Res. 150, 492-494 (1998).

23. Sasaki, M. S., Takatsuji, T. and Ejima, Y. The F Value Cannot be Ruled Out as a Chromosomal Fingerprint of Radiation Quality. Radiat. Res. 150, 253-258 (1998).

24. Lucas, J. N., Deng, W., Oram, S. W., Hill, F. S., Durante, M., George, K., Wu, H., Owens, C. L. and Yang, T. Theoretical and Experimental Tests of a Chromosomal Fingerprint for Densely Ionizing Radiation Based on F Ratios Calculated from Stable and Unstable Chromosome Aberrations. Radiat. Res. 151, 85-91 (1999).

25. Deng, W., Morrison, D. P., Gale, K. L. and Lucas, J. N. A Comparative Study on Potential Cytogenetic Fingerprints for Radiation LET in Human Lymphocytes. Int. J. Radiat. Biol. 76, 1589-1598 (2000).

26. Sachs, R. K., Brenner, D. J., Chen, A. M., Hahnfeldt, P. and Hlatky, L. R. Intra-arm and Interarm Chromosome Intrachanges: Tools for Probing the Geometry and Dynamics of Chromatin. Radiat. Res. 148, 330-340 (1997).

27. Lucas, J. N. and 10 others. Rapid Translocation Frequency Analysis in Humans Decades After Exposure to Ionizing Radiation. Int. J. Radiat. Biol. 62, 53-63 (1992).

28. Schrock, E. and 11 others. Multicolor Spectral Karyotyping of Human Chromosomes. Science 273, $494-497$ (1996).

29. Speicher, M. R., Gwyn Ballard, S. and Ward, D. C. Karyotyping Human Chromosomes by Combinatorial Multi-Fluor FISH. Nat. Genet. 12, 368-375 (1996).

30. Chudoba, I., Plesch, A., Lorch, T., Lemke, J., Claussen, U. and Senger, G. High Resolution Multicolor-Banding: A New Technique for Refined FISH Analysis of Human Chromosomes. Cytogenet. Cell Genet. 84, 156-160 (1999).

31. Muller, S., Rocchi, M., Ferguson-Smith, M. A. and Wienberg, J. Toward a Multicolor Chromosome Bar Code for the Entire Human Karyotype by Fluorescence in situ Hybridization. Hum. Genet. 100, 271-278 (1997).

32. Awa, A. A., Sofuni, T., Honda, T., Itoh, M., Neriishi, S. and Otake, M. Relationship Between the Radiation Dose and Chromosome Aberrations in Atomic Bomb Survivors of Hiroshima and Nagasaki. J. Radiat. Res. (Tokyo) 19, 126-140 (1978).

33. Ohtaki, K. G-Banding Analysis of Radiation-Induced Chromosome Damage in Lymphocytes of Hiroshima A-Bomb Survivors. Jpn J. Human Genet. 37, 245-262 (1992).

34. Sachs, R. K., Awa, A. A., Kodama, Y., Nakano, M., Ohtaki, K. and Lucas, J. N. Ratios of Radiation-Produced Chromosome Aberrations as Indicators of Large-Scale DNA Geometry During Interphase. Radiat. Res. 133, 345-350 (1993).

35. Anderson, R. M., Marsden, S. J., Wright, E. G., Kadhim, M. A., Goodhead, D. T. and Griffin, C. S. Complex Chromosome Aberrations in Peripheral Blood Lymphocytes as a Potential Biomarker of Exposure to High-LET Alpha-Particles. Int. J. Radiat Biol. 76, 31-42 (2000).

36. Pandita, T. K. and Geard, C. R. Chromosome Aberrations in Human Fibroblasts Induced by Monoenergetic Neutrons. I. Relative Biological Effectiveness. Radiat. Res. 145, 730-739 (1996).

37. Perera, F. P. and Weinstein, I. B. Molecular Epidemiology: Recent Advances and Future Directions. Carcinogenesis 21, 517-524 (2000).

38. Koshurnikova, N. A., Shilnikova, N. S., Okatenko, P. V., Kreslov, V. V., Bolotnikova, M. G., Sokolnikov, M. E., Khokhriakov, V. F., Suslova, K. G., Vassilenko, E. K. and Romanov, S. A. Characteristics of a Cohort of Workers at the Mayak Nuclear Complex. Radiat. Res. 152, 352-363 (1999). 
